\title{
CLASSIFYING RELATIVE EQUILIBRIA. II
}

\author{
BY JULIAN I. PALMORE ${ }^{1}$
}

Communicated by I. M. Singer, November 11, 1974

We announce several theorems which suggest a minimal classification of relative equilibria in the planar $n$-body problem. These theorems also answer several questions on the nature of degenerate relative equilibria classes which were asked recently by S. Smale [3]. A summary of previous results can be found in an earlier paper [1]. It is a pleasure to thank S. Smale for encouragement in this work.

1. Morse theory and relative equilibria. We study the critical set of a real analytic function $\widetilde{V}_{m}<0$ on a real analytic manifold $X_{m}$ where $n \geqslant 3$ and $m=\left(m_{1}, \ldots, m_{n}\right) \in R_{+}^{n}$ are fixed. Critical points of $\widetilde{V}_{m}$ correspond in a 1-1 fashion to classes of relative equilibria. $\widetilde{V}_{m}$ always has a compact critical set which we may investigate by Morse theory even when degenerate critical points exist [2].

The integral singular homology of $X_{m}$ (a manifold which is homeomorphic to a Stein manifold $\left.P_{n-2}(C)-\widetilde{\Delta}_{n-2}\right)$ is given by a recurrence relation [1] This suggests that there is a uniform lower bound on the number of critical points of each index of $\widetilde{V}_{m}$ which is given by recurrence. As a first step toward classifying relative equilibria Theorem 1 gives such a relation.

In Theorem 2 we assert that $\widetilde{V}_{m}$ is a Morse function for any $n \geqslant 3$ and for almost all $m \in R_{+}^{n}$ (in the sense of Lebesgue measure).

Theorem 3 answers the question: Is $\widetilde{V}_{m}$ always a Morse function?

Finally, we examine the case of four masses to show how a degeneracy of $\widetilde{V}_{m}$ arises. An interpretation of Theorem 1 in the degenerate case sheds light on the creation and annihilation of relative equilibria.

2. Main theorems. In this paragraph for any $i, 0 \leqslant i \leqslant 2 n-4$, let $\mu_{i}(n)$ denote a uniform lower bound to the number of critical points of $\widetilde{V}_{m}$

AMS (MOS) subject classifications (1970). Primary 70F10; Secondary 57D70.

Key words and phrases. Relative equilibria, Morse theory, $n$-body problem.

1 Research supported in part by NSF grant P-22930. 
with index equal to $2 n-4-i$ whenever $\widetilde{V}_{m}$ is a Morse function. By [1, Theorem 2] the index of any critical point of $\widetilde{V}_{m}$ whether degenerate or not has $n-2$ as a lower bound.

THEOREM 1. For any $n \geqslant 3$ and for any $i, 0 \leqslant i \leqslant n-2, \mu_{i}(n)=$ $(n-1-i) \mu_{i}(n-1)+(2 n-2-i) \mu_{i-1}(n-1)$; and $\mu_{i}(n)=0$ for $i>n-2$.

Corollary 1.1. $\mu_{i}(n)=C_{n, i}(n-1-i)(n-2) !$ for any $i, 0 \leqslant i \leqslant$ $n-2$, and for any $n \geqslant 3$.

Here $C_{n, i}$ is the binomial coefficient.

CoROllaRY 1.2. $\sum_{i=0}^{n-2} \mu_{i}(n)=\left[2^{n-1}(n-2)+1\right](n-2)$ ! for any $n \geqslant 3$.

Let $\beta_{i}=\operatorname{rank} H_{i}\left(P_{n-2}(C)-\widetilde{\Delta}_{n-2}\right)$ for any $i, 0 \leqslant i \leqslant 2 n-4$, and $n \geqslant 3$ where $H_{*}$ is the integral singular homology. We write $A(t)>B(t)$ for any two polynomials $A(t), B(t)$ provided that $A(t)-B(t)=(1+t) C(t)$ where $C(t)$ has nonnegative coefficients. This relation subsumes the Morse inequalities.

Corollary 1.3. $\Sigma_{i=0}^{n-2} \mu_{i}(n) t^{i}>\Sigma_{i=0}^{n-2} \beta_{i} t^{i}$ for any $n \geqslant 3$.

Recently, S. Smale [3] has raised questions about the nature of the set of masses $\Sigma_{n} \subset R_{+}^{n}$ on which degeneracies of $\widetilde{V}_{m}$ arise. The next two theorems give some measure-theoretic properties of $\Sigma_{n}$.

THEOREM 2. $\widetilde{V}_{m}$ is a Morse function for any $n \geqslant 3$ and for almost all masses $m \in R_{+}^{n}$ (in the sense of Lebesgue measure).

COROLlARY 2.1. There are only finitely many relative equilibria classes in the planar n-body problem for any $n \geqslant 3$ and for almost all masses $m \in R_{+}^{n}$.

REMARK. It is an open question whether for some $n \geqslant 4$ and $m \in R_{+}^{n}$ there are infinitely many critical points of $\widetilde{V}_{m}$.

Theorem 2 shows that $\Sigma_{n}$ has measure 0 for all $n \geqslant 3$. By [1, Theorem 4] we have $\Sigma_{3}=\varnothing$. The next result shows that for $n \geqslant 4$ degeneracies arise.

THEOREM 3. $\Sigma_{n} \neq \varnothing$ for any $n \geqslant 4$.

3. Classifying relative equilibria. For any three positive masses there are precisely five critical points of $\widetilde{V}_{m}$ and these critical points are nondegenerate. Their distribution corresponds to that of the minimal classification given by Theorem 1 .

For $n=4$ masses a degeneracy arises in the following fashion. In the 
plane $E^{2}$ we place three unit masses at the vertices of an equilateral triangle with center of mass at the origin. We place at the origin an arbitrary fourth positive mass, $m_{4}$. It follows easily for all values of $m_{4}$ that this configuration is a relative equilibrium.

Let $m=\left(1,1,1, m_{4}\right)$ and let $x \in X_{m}$ be the relative equilibria class to which the above relative equilibrium belongs. Let $D^{2} \widetilde{V}_{m}(x)$, the hessian of $\widetilde{V}_{m}$ at $x$, a real symmetric bilinear form on $T_{x} X_{m}$, be considered a function of $m_{4}$. By direct calculation [2] we find that the hessian is degenerate if and only if $m_{4}$ equals the unique positive number $m_{4}^{*}$ which is given by $m_{4}^{*}=$ $(2+3 \sqrt{3}) /(18-5 \sqrt{3})<1$.

For $m_{4}<m_{4}^{*}$ the index of $x$ (i.e. the index of the hessian of $\widetilde{V}_{m}$ at $x$ ) equals 4 and $x$ is a nondegenerate local maximum of $\widetilde{V}_{m}$. For $m_{4} \geqslant m_{4}^{*}$ the index of $x$ equals 2 . When $m_{4}=m_{4}^{*}$ the dimension of the nullspace of the hessian equals 2 . This is the maximum degeneracy possible for four masses.

These considerations suggest the following interpretation of Theorem 1 whenever $\widetilde{V}_{m}$ has isolated degenerate critical points.

For any $n \geqslant 4$ let $m \in R_{+}^{n}$ be such that $\widetilde{V}_{m}$ has only isolated critical points. Let $c_{1}<\ldots<c_{r}<0$ be the critical values of $\widetilde{V}_{m}$. Set $c_{0}=-\infty$ and for any $j, 1 \leqslant j \leqslant r$, define $W_{j}=\widetilde{V}_{m}^{-1}\left(c_{j-1}, c_{j}\right)$. Let $\Lambda_{j}$ be the set of critical points at level $j, 1 \leqslant j \leqslant r$. Finally, for any $i, 0 \leqslant i \leqslant 2 n-4$, define $\tau_{i}(n, m)$ by

$$
\tau_{i}(n, m)=\sum_{j=1}^{r} \operatorname{rank} H_{2 n-4-i}\left(W_{j} \cup \Lambda_{j}, W_{j}\right) .
$$

By $\left[1\right.$, Theorem 2] we have $\tau_{i}(n, m)=0$ for any $i>n-2$.

THEOREM 4. For any $n \geqslant 4$ and any $m \in R_{+}^{n}$ for which $\widetilde{V}_{m}$ has only isolated critical points, $\tau_{i}(n, m) \geqslant \mu_{i}(n)$ for any $i, 0 \leqslant i \leqslant 2 n-4$.

CoRollaRy 4.1. $\sum_{i=0}^{n-2} \tau_{i}(n, m) t^{i}>\sum_{i=0}^{n-2} \mu_{i}(n) t^{i}$ for any $n \geqslant 4$.

\section{REFERENCES}

1. J. I. Palmore, Classifying relative equilibria. I, Bull. Amer. Math. Soc. 79 (1973), 904-908. MR 47 \#9922.

2. Relative equilibria of the n-body problem, Thesis, University of California, Berkeley, Calif., 1973.

3. S. Smale, Problems in dynamical systems and celestial mechanics, (preprint). University of California, Berkeley, Calif., 1974.

DEPARTMENT OF MATHEMATICS, MASSACHUSETTS INSTITUTE OF TECHNOLOGY, CAMBRIDGE, MASSACHUSETTS 02139 